\title{
Suscetibilidade antimicrobiana de Staphylococcus spp. isolados de cães com pioderma superficial ${ }^{1}$
}

\author{
Ana Paula da Silva ${ }^{2 *}$, Claudete Schmidt ${ }^{3}$, Agueda C. de Vargas ${ }^{4}$, Grazieli \\ Maboni ${ }^{4}$, Carine Rampelotto ${ }^{4}$, Marcelo L. Schwab ${ }^{4}$, Talita P. Escobar ${ }^{3}$ \\ e Anne S. do Amaral ${ }^{3}$
}

\begin{abstract}
Silva A.P., Schmidt C., Vargas A.C., Maboni G., Rampelotto C., Schwab M.L., Escobar T.P. \& Amaral A.S. 2014. [Antimicrobial susceptibility of Staphylococcus spp. isolated from canine superficial pyoderma.] Suscetibilidade antimicrobiana de Staphylococcus spp. isolados de cães com pioderma superficial. Pesquisa Veterinária Brasileira 34(4):355-361. Hospital Veterinário, Departamento de Clínica de Pequenos Animais, Centro de Ciências Rurais, Universidade Federal de Santa Maria, Av. Roraima 1000, Prédio 97, Camobi, Santa Maria, RS 97105-100, Brazil. E-mail: apsvet@hotmail.com

A total of 154 samples of skin lesions were obtained from dogs with superficial pyoderma that were assisted by the Veterinary Dermatology Service at the University Veterinary Hospital (HVU), Universidade Federal de Santa Maria (UFSM), aiming to determine the antimicrobial susceptibility of Staphylococcus spp. isolates and evaluate the presence of multidrug resistance profile. After bacterial isolation and identification, the strains were tested for antimicrobial susceptibility, and the results showed lower percentages of resistance to the amoxicillin and clavulanic acid association (1.9\%), cefadroxil (1.9\%), cephalexin $(1.9 \%)$ and vancomycin $(0.6 \%)$. The highest percentages were towards amoxicillin $(60.4 \%)$ and penicillin $\mathrm{G}(60.4 \%)$. The multidrug resistance was detected in $23.4 \%$ and the methicillin resistance in $5.8 \%$ of the samples. It may be concluded that the Staphylococcus spp. isolates present high susceptibility to key antimicrobials used in the treatment of superficial pyodermas in dogs at the HVU-UFSM, such as cephalexin and the amoxicillin and clavulanic acid association, confirming the preference for these drugs when treating dogs with this disorder. The susceptibility of the strains to fluoroquinolones, also recommended in the literature for the treatment of pyodermas, allows suggesting that such drugs should not be considered in the empirical selection. The identification of multidrug-resistant Staphylococcus spp. in the studied canine population justifies periodic and regional bacteriological tests of skin lesions in dogs with superficial pyoderma, possible therapeutic failures and also motivates wise use of antimicrobial therapy.
\end{abstract}

INDEX TERMS: Diseases of dogs, dermatology, skin infection, antimicrobials, bacterial resistance.

RESUMO.- Foram obtidas 154 amostras de lesões cutâneas de cães com pioderma superficial atendidos no Serviço

\footnotetext{
${ }^{1}$ Recebido em 3 de outubro de 2013.

Aceito para publicação em 30 de dezembro de 2013.

${ }^{2}$ Programa de Pós-Graduação em Medicina Veterinária (PPGMV), Universidade Federal de Santa Maria (UFSM), Camobi, Santa Maria, RS 97105100, Brasil. *Autor para correspondência: apsvet@hotmail.com

${ }^{3}$ Hospital Veterinário Universitário (HVU), Departamento de Clínica de Pequenos Animais (DCPA), UFSM, Santa Maria, RS 97105-100. E-mails: dete 13vet@yahoo.com.br, talitapescobar@gmail.com, anne.am@hotmail.com

${ }^{4}$ Departamento de Medicina Veterinária Preventiva, Centro de Ciências Rurais, UFSM, Santa Maria, RS 97105-900. E-mails: agueda.vargas@ gmail.com, grazimaboni@hotmail.com, ninerampelotto@gmail.com, marschwab17@gmail.com
}

de Dermatologia Veterinária do Hospital Veterinário Universitário (HVU) da Universidade Federal de Santa Maria (UFSM), com o objetivo de determinar a suscetibilidade antimicrobiana de isolados de Staphylococcus spp. e avaliar a presença de multirresistência. Após isolamento e identificação, as cepas foram submetidas ao teste de sensibilidade aos antimicrobianos, cujos resultados evidenciaram menores percentuais de resistência à associação amoxicilina e ácido clavulânico $(1,9 \%)$, cefadroxil $(1,9 \%)$, cefalexina $(1,9 \%)$ e vancomicina $(0,6 \%)$. Os maiores percentuais de resistência foram frente à amoxicilina $(60,4 \%)$ e penicilina G $(60,4 \%)$. A multirresistência foi detectada em $23,4 \%$ e a resistência à meticilina em 5,8\% das amostras. Pode-se 
concluir que os isolados de Staphylococcus spp. apresentam elevada suscetibilidade aos antimicrobianos comumente utilizados no tratamento dos piodermas superficiais em cães atendidos no HVU-UFSM, como a cefalexina e a amoxicilina associada ao ácido clavulânico, confirmando a eleição desses fármacos para o tratamento de cães com esta afecção. A suscetibilidade diminuída das cepas frente às fluoroquinolonas, também recomendadas pela literatura para o tratamento de pioderma, permite sugerir que estes fármacos não devem mais ser considerados na seleção empírica. A identificação de Staphylococcus spp. multirresistentes na população canina estudada justifica análises bacteriológicas periódicas e regionais de lesões cutâneas de cães com pioderma superficial, a fim de minimizar a seleção de bactérias resistentes, possíveis falhas terapêuticas e também motiva a antimicrobianoterapia prudente.

TERMOS DE INDEXAÇÃO: Doenças de cães, dermatologia, infecção cutânea, antimicrobianos, resistência bacteriana.

\section{INTRODUÇÃO}

Pioderma (ou piodermite) superficial é definido como a infecção bacteriana do tegumento que envolve o folículo piloso e a epiderme adjacente (Scott et al. 2001) e representa uma das dermatopatias mais frequentes em cães (Hill et al. 2006, Souza et al. 2009, Hnilica 2011). Os principais agentes etiológicos são bactérias do gênero Staphylococcus (Cox 2006, Ihrke 2006), sendo Staphylococcus pseudintermedius (S. pseudintermedius) anteriormente referido como S. intermedius (Mason 1991, Hill \& Moriello 1994, Scott et al. 2001), a espécie mais comumente isolada (Gross et al. 2005, Devriese et al. 2009, Hnilica 2011). Na maioria dos casos, a piodermite superficial é considerada uma manifestação secundária de outras alterações cutâneas ou sistêmicas e os animais acometidos podem apresentar quadros recorrentes dessa infecção como uma importante complicação da doença subjacente (Scott et al. 2001).

Pioderma constitui uma das principais causas das indicações de antimicrobianoterapia em cães, o que, geralmente, é realizado de forma empírica, sendo as cefalosporinas os fármacos de primeira escolha para o tratamento (Ihrke 2005, May 2006, Guardabassi et al. 2008, Hnilica 2011). Adicionalmente, o pioderma superficial pode ocorrer de forma crônica e recidivante, fazendo-se necessário o uso frequente ou prolongado de antimicrobianos (Scott et al. 2001, Ihrke 2006). Tais fatores predispõem à seleção de bactérias resistentes e/ou multirresistentes e consequentes falhas terapêuticas, o que torna difícil o manejo dessa afecção (Guardabassi et al. 2008).

Diversos autores evidenciaram, nos últimos anos, significativo aumento da prevalência de Staphylococcus spp. multirresistentes e resistentes à meticilina (MRS) em medicina veterinária (Jones et al. 2007, Duijkeren et al. 2011, Nienhoff et al. 2011, Bond \& Loeffler 2012). A emergência desses patógenos, isolados principalmente de lesões cutâneas, otites e infecções pós-cirúrgicas, implica em um grave problema devido à dificuldade no tratamento e ao considerável potencial zoonótico (Duquette \& Nuttall 2004, Guardabassi et al. 2008).
Estudos de suscetibilidade antimicrobiana de Staphylococcus spp. apontaram amplas variações nos resultados dependendo da área geográfica analisada (Guardabassi et al. 2008). Embora existam pesquisas relacionadas ao perfil antimicrobiano desses patógenos em cães com piodermas no Brasil (Cavalcanti \& Coutinho 2005, Pianta et al. 2006), dados atuais referentes à prevalência de multirresistência ainda são escassos (Penna et al. 2009, Lima et al. 2012).

A ampla utilização de antimicrobianos na terapia da piodermite canina, a emergência de cepas resistentes e as possíveis variações quanto à suscetibilidade antimicrobiana dos Staphyloccocus spp., despertou o interesse em investigar esses achados em cães com pioderma superficial.

Diante disso, o objetivo deste estudo foi determinar a suscetibilidade antimicrobiana e avaliar a presença de multirresistência em cepas de Staphylococcus spp. oriundas de lesões cutâneas de cães com pioderma superficial.

\section{MATERIAL E MÉTODOS}

Foram avaliados 154 isolados bacterianos provenientes de lesões cutâneas em cães com pioderma superficial, atendidos pelo Serviço de Dermatologia do Hospital Veterinário Universitário (HVU) da Universidade Federal de Santa Maria (UFSM), durante o ano de 2012. Cães apresentando colaretes epidérmicos, com diagnóstico firmado pelo mesmo clínico e que não haviam sido submetidos à terapia antimicrobiana prévia (no mínimo 30 dias antes da avaliação clínica) foram incluídos no estudo, independente da idade, sexo ou raça. As amostras foram acondicionadas em frascos estéreis contendo meio de transporte de Stuart e imediatamente encaminhadas para análise no Laboratório de Bacteriologia (LABAC) da mesma instituição.

O cultivo bacteriano foi realizado pela técnica de semeadura por esgotamento, em ágar sangue ovino a 5\%, seguido de incubação a $37^{\circ} \mathrm{C}$ por 24 horas. Na sequência, foi realizada a identificação das colônias segundo as características morfo-tintoriais e bioquímicas (Carter 1988). A análise bacteriológica baseou-se na morfologia, pigmentação das colônias, tipo de hemólise, coloração de Gram, reação da catalase, coagulase (em lâmina e em tubo), comportamento frente à polimixina $\mathrm{B}$, além de testes bioquímicos de ONPG (orto-nitrofenil-galactopiranosídeo) e fermentação da maltose. Como não foram utilizados métodos moleculares para diferenciação das espécies de Staphylococcus e as análises bioquímicas tradicionais podem apresentar falhas, aqueles foram referidos como Staphylococcus spp.

Após a identificação dos isolados, esses foram submetidos ao teste de suscetibilidade aos antimicrobianos (TSA) através do método de disco-difusão em ágar, descrito por Bauer et al. (1966). Para tanto, foi realizado um inóculo de suspensão direta de colônias bacterianas oriundas de ágar sangue ovino $5 \%$, obtendo-se turbidez de 0,5 na escala de McFarland. 0 inóculo foi semeado em ágar Mueller-Hinton e discos de antimicrobianos foram depositados em sua superfície, com as placas incubadas a $35^{\circ} \mathrm{C}$, por 24 horas. Foram testados os seguintes antimicrobianos pertencentes às classes: 1) betalactâmicos: a) família das penicilinas - amoxacilina $(10 \mu \mathrm{g})$, oxacilina $(1 \mu \mathrm{g})$, penicilina $\mathrm{G}(10 \mathrm{U})$, amoxicilina com ácido clavulânico $(30 \mu \mathrm{g}), \mathrm{b})$ família das cefalosporinas - cefadroxil $(30 \mu \mathrm{g})$, cefalexina $(30 \mu \mathrm{g}) ; 2)$ macrolídeos - azitromicina $(15 \mu \mathrm{g})$; $3)$ fluoroquinolonas - enrofloxacina $(5 \mu \mathrm{g})$, ciprofloxacina $(5 \mu \mathrm{g})$; 4) tetraciclinas - doxiciclina $(30 \mu \mathrm{g}) ; 5)$ polimixinas - polimixina B

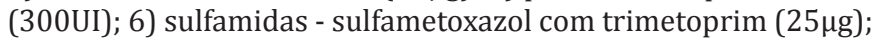
7) glicopeptídeos - vancomicina $(30 \mu \mathrm{g})$. A escolha dos antimicrobianos baseou-se em indicações terapêuticas de May (2006) e 
Hnilica (2011), com exceção da polimixina B, usada na diferenciação fenotípica de espécies e da oxacilina, testada para avaliar a suscetibilidade à meticilina. Cepas de referência de Staphylococcus aureus ATCC 25923 foram utilizadas no controle de qualidade do método de disco-difusão e os resultados dos testes interpretados segundo as normas do "Clinical Laboratory Standards Institute" (CLSI 2006). Os isolados foram classificados em sensíveis, intermediários ou resistentes aos antimicrobianos (considerando os intermediários como resistentes), mediante a leitura da medida dos halos de inibição de crescimento bacteriano e, ao final dos procedimentos, liofilizados e estocados a $-20^{\circ} \mathrm{C}$.

A multirresistência foi definida como resistência a três ou mais classes de antimicrobianos, conforme Youn et al. (2011). A resistência à meticilina foi avaliada pelo método de suscetibilidade em disco-difusão utilizando-se oxacilina. Para comparar a resistência entre os antimicrobianos testados foi realizada análise estatística dos dados através do teste Qui-quadrado com nível de significância $\mathrm{P}<0,05$, utilizando-se o programa SAS.

\section{RESULTADOS}

Quanto aos percentuais de sensibilidade dos 154 isolados de Staphylococcus spp. aos 13 antimicrobianos testados neste estudo, foram encontrados os seguintes resultados: amoxicilina $39,6 \%$, oxacilina $94,2 \%$, penicilina $39,6 \%$, amoxicilina/clavulanato de potássio 98,1\%, cefadroxil 98,1\%, cefalexina $98,1 \%$, azitromicina $82,5 \%$, enrofloxacina $79,9 \%$, ciprofloxacina $81,2 \%$, doxiciclina $95,5 \%$, polimixina B 98,1\%, sulfametoxazol/trimetoprim 70,1\% e vancomicina $99,4 \%$. Em relação à resistência, os valores foram amoxicilina $60,4 \%$, oxacilina $5,8 \%$, penicilina $60,4 \%$, amoxicilina/ clavulanato de potássio $1,9 \%$, cefadroxil $1,9 \%$, cefalexina $1,9 \%$, azitromicina $17,5 \%$, enrofloxacina $20,1 \%$, ciprofloxacina $18,8 \%$, doxiciclina $4,5 \%$, polimixina B $1,90 \%$, sulfametoxazol/trimetoprim $29,9 \%$ e vancomicina $0,6 \%$ (Fig. 1).

Levando em consideração as classes de antimicrobianos, os maiores percentuais de resistência foram no grupo das sulfamidas $29,9 \%(46 / 154)$, seguido pelos betalactâmi$\cos 23,76 \%$ (13/154), fluoroquinolonas 22,34\% (12/154)

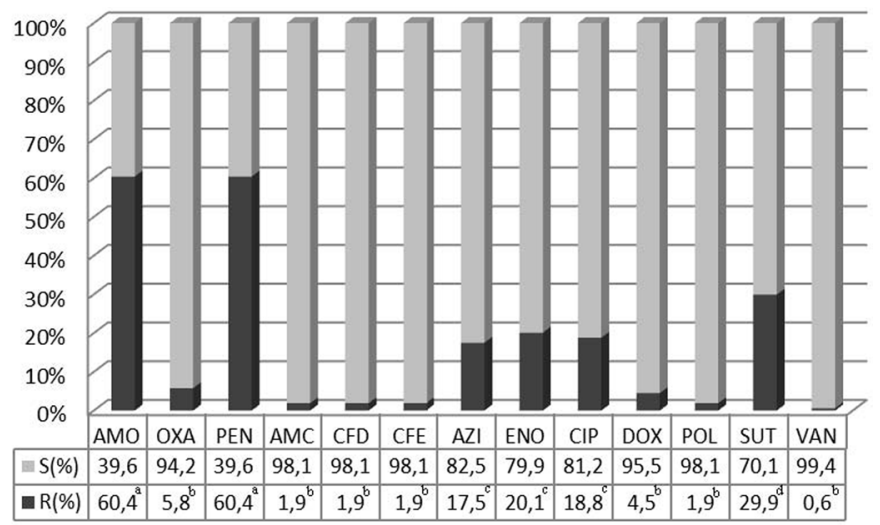

Fig.1. Suscetibilidade aos antimicrobianos de 154 Staphylococcus spp. isolados de cães com pioderma superficial. $\mathrm{S}=$ sensibilidade (\%); $\mathrm{R}=$ resistência $(\%) ; \mathrm{AMO}=$ amoxicilina; $\mathrm{OXA}=$ oxacilina; $\mathrm{PEN}=$ penicilina; $\mathrm{AMC}=$ amoxicilina/clavulanato de potássio; $\mathrm{CFD}$ = cefadroxil; $\mathrm{CFE}$ = cefalexina; $\mathrm{AZI}=$ azitromicina; $\mathrm{ENO}=$ enrofloxacina; $\mathrm{CIP}$ = ciprofloxacina; DOX = doxiciclina; $\mathrm{POL}=$ polimixina $\mathrm{B} ; \mathrm{SUT}=$ sulfametoxazol/trimetoprim; VAN = vancomicina; $\mathrm{a}, \mathrm{b}, \mathrm{c}, \mathrm{d}=$ percentagens com letras diferentes diferem entre si pelo teste do qui-quadrado $(\mathrm{P}<0,05)$. e macrolídeos $17,5 \%(27 / 154)$. Os menores percentuais de resistência detectados foram na classe das tetraciclinas $4,5 \%$ (7/154), polimixinas 1,9\% (3/154) e glicopeptídeos $0,6 \%(1 / 154)$.

Quanto à multirresistência, 23,4\% (36/154) dos Staphylococcus spp. analisados apresentaram este padrão sendo que, dentre os multirresistentes, 24 cepas apresentaram resistência a três classes de antimicrobianos, 11 cepas a quatro classes e uma cepa frente a cinco classes (Quadro 1).

\section{Quadro 1. Suscetibilidade aos antimicrobianos de 154 Staphylococcus spp. isolados de cães com pioderma superficial}

\begin{tabular}{|c|c|c|}
\hline Perfil de Resistência & & tal (\%) \\
\hline $\mathrm{AMO}^{1}$ & 1 & $0,65 \%$ \\
\hline CIP $^{1}$ & 1 & $0,65 \%$ \\
\hline $\mathrm{ENO}^{1}$ & 1 & $0,65 \%$ \\
\hline PEN $^{1}$ & 2 & $1,30 \%$ \\
\hline SUT $^{1}$ & 2 & $1,30 \%$ \\
\hline $\mathrm{AMO}+\mathrm{AMC}^{1}$ & 1 & $0,65 \%$ \\
\hline $\mathrm{AMO}^{-\mathrm{PEN}^{1}}$ & 32 & $20,78 \%$ \\
\hline $\mathrm{CEF}+\mathrm{AZI}^{2}$ & 1 & $0,65 \%$ \\
\hline $\mathrm{DOX}+\mathrm{SUT}^{2}$ & 2 & $1,30 \%$ \\
\hline $\mathrm{ENO}+\mathrm{CIP}^{1}$ & 1 & $0,65 \%$ \\
\hline $\mathrm{OXA}+\mathrm{AZI}^{2}$ & 1 & $0,65 \%$ \\
\hline $\mathrm{PEN}+\mathrm{AMC}^{1}$ & 1 & $0,65 \%$ \\
\hline $\mathrm{AMO}+\mathrm{ENO}+\mathrm{CIP}^{2}$ & 1 & $0,65 \%$ \\
\hline $\mathrm{AMO}+\mathrm{OXA}+\mathrm{PEN}^{1}$ & 2 & $1,30 \%$ \\
\hline $\mathrm{AMO}+\mathrm{PEN}+\mathrm{AZI}^{2}$ & 5 & $3,25 \%$ \\
\hline $\mathrm{AMO}+\mathrm{PEN}+\mathrm{CFE}^{1}$ & 1 & $0,65 \%$ \\
\hline $\mathrm{AMO}+\mathrm{PEN}+\mathrm{DOX}^{2}$ & 3 & $1,95 \%$ \\
\hline $\mathrm{AMO}+\mathrm{PEN}+\mathrm{ENO}^{2}$ & 1 & $0,65 \%$ \\
\hline $\mathrm{AMO}+\mathrm{PEN}+\mathrm{POL}^{2}$ & 1 & $0,65 \%$ \\
\hline $\mathrm{AMO}+\mathrm{PEN}+\mathrm{SUT}^{2}$ & 8 & $5,19 \%$ \\
\hline $\mathrm{OXA}+\mathrm{ENO}+\mathrm{POL}^{3^{*}}$ & 1 & $0,65 \%$ \\
\hline $\mathrm{AMO}+\mathrm{OXA}+\mathrm{PEN}+\mathrm{AZI}^{2}$ & 1 & $0,65 \%$ \\
\hline $\mathrm{AMO}+\mathrm{PEN}+\mathrm{AZI}+\mathrm{SUT}^{3^{*}}$ & 7 & $4,55 \%$ \\
\hline $\mathrm{AMO}+\mathrm{PEN}+\mathrm{CIP}+\mathrm{SUT}^{3^{*}}$ & 2 & $1,30 \%$ \\
\hline $\mathrm{AMO}+\mathrm{PEN}+\mathrm{DOX}+\mathrm{SUT}^{3 *}$ & 1 & $0,65 \%$ \\
\hline $\mathrm{AMO}+\mathrm{PEN}+\mathrm{ENO}+\mathrm{POL}^{3^{*}}$ & 1 & $0,65 \%$ \\
\hline $\mathrm{AMO}+\mathrm{PEN}+\mathrm{OXA}+\mathrm{VAN}^{2}$ & 1 & $0,65 \%$ \\
\hline $\mathrm{AMO}+\mathrm{PEN}+\mathrm{ENO}+\mathrm{CIP}+\mathrm{SUT}^{3^{*}}$ & 12 & $7,79 \%$ \\
\hline $\mathrm{AMO}+\mathrm{PEN}+\mathrm{AZI}+\mathrm{ENO}+\mathrm{CIP}+\mathrm{SUT}^{4 *}$ & 6 & $3,90 \%$ \\
\hline $\mathrm{AMO}+\mathrm{PEN}+\mathrm{AZI}+\mathrm{ENO}+\mathrm{CIP}+\mathrm{DOX}+\mathrm{SUT}^{5^{*}}$ & 1 & $0,65 \%$ \\
\hline $\mathrm{AMO}+\mathrm{OXA}+\mathrm{PEN}+\mathrm{CEF}+\mathrm{AZI}+\mathrm{ENO}+\mathrm{CIP}+\mathrm{SUT}^{4^{*}}$ & 4 & $2,60 \%$ \\
\hline $\mathrm{AMO}+\mathrm{OXA}+\mathrm{PEN}+\mathrm{AMC}+\mathrm{CFD}+\mathrm{CFE}+\mathrm{AZI}+\mathrm{ENO}+\mathrm{CIP}+\mathrm{SUT}^{4^{*}}$ & 1 & $0,65 \%$ \\
\hline Sem resistência & 48 & $31,17 \%$ \\
\hline TOTAL & 154 & $100 \%$ \\
\hline
\end{tabular}

AMO = amoxicilina; $\mathrm{OXA}=$ oxacilina PEN $=$ penicilina AMC $=$ amoxici lina/clavulanato de potássio; $\mathrm{CFD}=$ cefadroxil; $\mathrm{CFE}=$ cefalexina; $\mathrm{AZI}=$ azitromicina; $\mathrm{ENO}=$ enrofloxacina; $\mathrm{CIP}=$ ciprofloxacina; $\mathrm{DOX}=$ doxiciclina; $\mathrm{POL}=$ polimixina $\mathrm{B} ; \mathrm{SUT}=$ sulfametoxazol/trimetoprim; $\mathrm{VAN}=$ vancomicina; 1 = resistência a uma classe; 2 = resistência a duas classes; $3=$ resistência a três classes; $4=$ resistência a quatro classes; $5=$ resistência a cinco classes; *multirresistentes.

\section{DISCUSSÃO}

Os percentuais de sensibilidade e resistência dos 154 isolados de Staphylococcus spp. aos 13 antimicrobianos podem ser visualizados na Figura 1. Pode-se verificar que, na classe dos betalactâmicos, mais de $50 \%$ dos isolados demonstraram resistência à família das penicilinas, com $60,4 \%$ (93/154) resistentes à amoxicilina e também à penicilina $\mathrm{G}$, enquanto que, menos de $2 \%$ dos isolados foram resis- 
tentes à cefalexina e cefadroxil, representantes da família das cefalosporinas, ambos com percentual de resistência de 1,9\% (3/154). 0 mesmo percentual de resistência, 1,9\% (3/154), foi observado frente à associação amoxicilina e ácido clavulânico. A alta resistência às penicilinas detectada neste estudo já havia sido citada em diversas pesquisas (Ganiere et al. 2005, Pedersen et al. 2007, Penna et al. 2009, Onuma et al. 2011, Proietti et al. 2012). Este resultado era esperado devido à produção de $\beta$-lactamases por grande parte dos Staphylococcus spp., as quais hidrolisam o núcleo ativo desses fármacos. Tal mecanismo de resistência às penicilinas explica a diferença entre os valores detectados frente à amoxicilina isoladamente $(60,4 \%)$ e, quando associada ao ácido clavulânico (1,9\%), uma vez que este ácido é um inibidor das $\beta$-lactamases produzidas pelos estafilococos (Spinosa et al. 2002).

Apesar de pertencer à classe dos betalactâmicos, as cefalosporinas são antimicrobianos estáveis às $\beta$-lactamases e, dessa forma, eficazes contra Staphylococcus spp. (Spinosa et al. 2002). Toma et al. (2008) consideraram incomum a resistência à cefalexina, antimicrobiano de primeira escolha para o tratamento do pioderma superficial em cães, fato confirmado neste estudo, no qual 98\% (151/154) das cepas demonstraram sensibilidade à cefalexina e cefadroxil. Resultados semelhantes foram observados em estudos conduzidos na França (Ganiere et al. 2005), Estados Unidos (White et al. 2005) e Itália (Vanni et al. 2009), onde os pesquisadores não detectaram resistência à cefalexina ao avaliar amostras de cães acometidos por pioderma. No Brasil, Cavalcanti \& Coutinho (2005) também encontraram $100 \%$ de isolados sensíveis à cefalexina, enquanto Pianta et al. (2006) averiguaram 93\% de sensibilidade a esse antimicrobiano. Por outro lado, em um recente estudo italiano, $54,3 \%$ e $50 \%$ dos $S$. pseudintermedius foram resistentes à cefalexina e cefadroxil, respectivamente (Proietti et al. 2012). Onnuma et al. (2011) compararam o perfil de resistência de $S$. pseudintermedius oriundos da pele de cães no Japão, em dois períodos distintos. Dos isolados obtidos entre 2000-2002, todos foram sensíveis à cefalexina, outras cefalosporinas e à oxacilina ao passo que, nas amostras coletadas em 2009 , obtiveram $12,5 \%$ de resistência às cefalosporinas e 11,4\% à oxacilina. Esses resultados são um alerta da possibilidade de mudança dos perfis de suscetibilidade, especialmente pela emergência de bactérias resistentes à meticilina, as quais frequentemente apresentam resistência cruzada às cefalosporinas.

A resistência à meticilina, que é utilizada para avaliar a multirresistência aos betalactâmicos, foi pesquisada neste estudo pelo método de disco-difusão e detectada em 5,8\% (9/154) dos isolados, resultado inferior aos de Jones et al. (2007), Nienhoff et al. (2011), Youn et al. (2011) e Proietti et al. (2012), nos quais a frequência de $S$. pseudintermedius resistentes à meticilina (MRSP) variou de 7,4\% a 41,4\% das amostras avaliadas. Como o aumento da prevalência de MRSP em cães com pioderma superficial é um acontecimento relativamente recente (Jones et al. 2007, Loeffler et al. 2007, Lucia et al. 2011), torna-se importante a avaliação local e periódica dos perfis de suscetibilidade. Como já mencionado, as cefalosporinas são os antimicrobianos de primeira escolha e utilizados com maior frequência no tratamento dessa dermatopatia, entretanto, frente a infecções cutâneas por MRSP, as cefalosporinas deixam de constituir opções terapêuticas, o que restringe a seleção dos fármacos.

Quanto à associação sulfametoxazol e trimetoprim, alguns estudos revelaram baixa resistência à essa combinação, como o realizado por Pedersen et al. (2007) que identificaram apenas $3,6 \%$ de isolados de estafilococos resistentes às sulfonamidas potencializadas em cães com pioderma na Dinamarca. Ganiere et al. (2005) não observaram isolados resistentes na mesma situação na França. Em contrapartida, em pesquisas conduzidas na Coreia (Youn et al. 2011) e na Itália (Proietti et al. 2012) foram detectadas $62,6 \%$ e $97,1 \%$ de amostras resistentes, respectivamente. Neste trabalho, a resistência frente à associação sulfametoxazol e trimetoprim foi de 29,9\% (46/154). Tais resultados se justificam, provavelmente, aos diferentes níveis de exposição da população canina aos antimicrobianos, conforme cada região. 0 emprego habitual desse fármaco no tratamento de cães acometidos por pioderma pela primeira vez pode ter auxiliado na seleção de bactérias resistentes às sulfonamidas ao longo dos anos (Ganiere et al. 2005). May (2006) caracterizou as sulfonamidas potencializadas como uma razoável opção terapêutica contra infecções causadas por estafilococos em pequenos animais, contudo, salientou que isolados resistentes à meticilina tendem a permanecer sensíveis a esta associação. Em virtude disso, deve haver prudência na sua utilização em dermatologia veterinária, pois podem constituir uma das poucas opções para o tratamento frente a cepas resistentes à meticilina.

Segundo May (2006), antimicrobianos da classe das fluoroquinolonas constituem boas escolhas no tratamento do pioderma canino o que está de acordo com os resultados de Pedersen et al. (2007), que obtiveram somente 1,2\% de resistência à enrofloxacina em 84 amostras de Staphylococcus spp. avaliadas. Os mesmos autores citaram o uso da ciprofloxacina, no entanto, pelo fato de não ser licenciado para uso em animais na Dinamarca, assim como em outros países, esse fármaco não é incluso nos testes. No mesmo estudo comparativo realizado por Onuma et al. (2011), todas as cepas isoladas de cães com pioderma foram suscetíveis às fluoroquinolonas no primeiro período avaliado, ao passo que, na década seguinte, $50 \%$ das cepas analisadas foram resistentes a tais fármacos. Ainda mais alarmantes foram os resultados de Proietti et al. (2012), que detectaram resistência à enrofloxacina em 94,3\% dos isolados. Neste estudo foram observados 20,1\% (31/154) e 18,8\% (29/154) de isolados resistentes à enrofloxacina e ciprofloxacina, respectivamente. Estes achados podem ser atribuídos à utilização frequente e indiscriminada dessa classe de antimicrobianos tanto na terapia de dermatopatias, como de outras infecções, principalmente enrofloxacina, a qual, no Brasil, é facilmente adquirida em estabelecimentos veterinários, sem necessidade de receituário. Para alguns autores, as fluoroquinolonas somente devem ser utilizadas em casos de piodermites por Staphylococcus spp. quando outros antimicrobianos tenham sido contraindicados ou ineficientes (Guardabassi et al. 2008, Patel \& Forsythe 2010), além de seu uso estar provavelmente relacionado 
ao aumento do risco de seleção de resistência à meticilina (Hnilica 2011).

Os antimicrobianos da classe dos macrolídeos podem ser empregados no tratamento de cães com pioderma superficial (Ihrke 2006) e foram considerados como primeira opção terapêutica por Patel e Forsythe (2010). Já Hnilica (2011) recomendou o seu uso somente quando a resposta aos antimicrobianos de primeira opção tenha sido insatisfatória. Entre os macrolídeos utilizados nas doenças bacterianas da pele em cães, existem poucos dados a respeito da eficácia da azitromicina (May 2006). Neste estudo, 17,5\% (27/154) dos isolados foram resistentes a este antimicrobiano. Pesquisas semelhantes, investigando a suscetibilidade à eritromicina apontaram índices de resistência superiores a 30\% (Ganiere et al. 2005, Pedersen et al. 2007). No Brasil, o maior percentual de resistência dos estafilococos isolados de cães com piodermite foi à eritromicina (55\%) (Cavalcanti \& Coutinho 2005) e à azitromicina (84\%) (Pianta et al. 2006), corroborando com Ihrke (2006) que considerou a resistência bacteriana aos macrolídeos relativamente comum.

A resistência antimicrobiana do $S$. pseudintermedius às tetraciclinas tem sido documentada na literatura entre as mais prevalentes. Em virtude disso, tais compostos tornaram-se, praticamente, sem valor terapêutico nos piodermas (Scott et al. 2001). Proietti et al. (2012) relataram 95,7\% de $S$. pseudintermedius resistentes à doxiciclina em um total de 70 amostras e Lima et al. (2012) detectaram 46,4\% de resistência à tetracilina, a qual, juntamente com as penicilinas, foi apontada entre as maiores taxas de resistência. Diferente dos resultados acima citados, neste estudo, o percentual de isolados resistentes à doxiciclina foi de apenas $4,5 \%$ (7/154), coincidindo com os resultados de Ganiere et al. (2005) e Ghidini et al. (2011) que encontraram 6\% e $10 \%$ de resistência a este fármaco, respectivamente. Os primeiros observaram ainda $48 \%$ dos isolados resistentes à oxitetraciclina e salientaram que, mesmo comprovando a resistência cruzada entre oxitetraciclina e doxiciclina, obtiveram baixa resistência à doxiciclina. Apesar do uso das tetraciclinas não ser preconizado no tratamento do pioderma canino (Scott et al. 2001, Hnilica \& May 2004, Hnilica 2011), Horvath \& Neuber (2007) e Guardabassi et al. (2008) incluíram a doxiciclina entre as opções de antimicrobianos sistêmicos para uso em cães com a doença, o que também pode ser recomendado após este estudo, levando em consideração o baixo percentual de resistência (Fig.1).

A vancomicina é um fármaco de importância terapêutica na medicina humana, especialmente em infecções por estafilococos multirresistentes. Já em medicina veterinária, seu uso além de não ser comum, somente é recomendado caso a infecção traga risco de vida e os testes de suscetibilidade tenham mostrado resistência a todos os outros antimicrobianos disponíveis (Guardabassi et al. 2008). Apenas uma das 154 amostras deste estudo foi resistente à vancomicina. A elevada sensibilidade $(99,4 \%)$ pode ser explicada pela utilização restrita desse antimicrobiano em animais de companhia e foi semelhante aos resultados de Youn et al. (2011) que não evidenciaram resistência à vancomicina entre 230 isolados de Staphylococcus spp. Entre- tanto, existem relatos de cepas clínicas de Staphylococcus spp. resistentes a todas as formulações orais de antimicrobianos disponíveis para o tratamento de pioderma e otites (Loeffler et al. 2007). A terapia antimicrobiana contra esses isolados torna-se crítica em animais, uma vez que, além da vancomicina, outros compostos empregados em humanos como linezolida, estreptograminas e tigeciclina são caros e todos, exceto linezolida, devem ser administrados por via intravenosa (Guardabassi et al. 2008).

Levando em consideração as classes de antimicrobianos, ocorreu maior resistência ao grupo das sulfamidas 29,9\% (46/154), seguido pelos betalactâmicos 23,76\% (13/154), fluoroquinolonas 22,34\% (12/154) e macrolídeos $17,5 \%$ (27/154). Os menores percentuais de resistência foram detectados às tetraciclinas $4,5 \%(7 / 154)$, polimixinas $1,9 \%$ (3/154) e glicopeptídeos $0,6 \%(1 / 154)$. Entretanto, quando se avalia separadamente a família das penicilinas e cefalosporinas, ambas pertencentes à classe dos betalactâmicos, na primeira evidenciaram-se os maiores percentuais de resistência enquanto na segunda, os menores (Fig.1). A análise estatística comprovou que os valores de resistência antimicrobiana diferiram entre os grupos das penicilinas e cefalosporinas $(\mathrm{P}<0,05)$. Dessa forma, a comparação da suscetibilidade entre classes torna-se arriscada, devido ao diferente número de antimicrobianos testados em cada classe e à ausência de homogeneidade dentro de uma mesma classe, como supracitado no caso dos betalactâmicos, em que há uma subdivisão em famílias de acordo com as suas propriedades farmacológicas. Portanto, neste estudo, o agrupamento dos antimicrobianos em classes, teve como principal objetivo identificar os isolados multirresistentes, sendo considerados aqueles resistentes a três ou mais classes de antimicrobianos.

Quanto à multirresistência, 23,4\% (36/154) dos Staphylococcus spp. analisados apresentaram esse padrão. Esses resultados foram semelhantes aos obtidos no estudo de Lima et al. (2012), cujo percentual de cepas multirresistentes foi de 34,2\% e, contrastaram com 77,1\% de multirresistência averiguada por Penna et al. (2009) e 73,0\% por Youn et al. (2011). Dentre os multirresistentes, em 24 verificou-se resistência a três classes de antimicrobianos, em 11 a quatro classes e um isolado frente a cinco classes (Quadro 1). Embora a multirresistência tenha íntima associação com estafilococos resistentes à meticilina em humanos e animais, esse padrão pode ocorrer na ausência de resistência à oxacilina (Jones et al. 2007), fato observado neste estudo, no qual cinco dos 36 isolados multirresistentes, também foram resistentes à oxacilina. Onuma et al. (2011) relataram expressiva elevação na prevalência de multirresistência entre $S$. pseudintermedius isolados de cães com pioderma no Japão, entre 1999 e 2009. Os autores atribuíram esse aumento ao uso indiscriminado de antimicrobianos e relataram ter sido mais marcado em cães submetidos à antimicrobianoterapia prévia. Neste estudo, dos 36 isolados de Staphylococcus spp. multirresistentes, em 29 casos os cães haviam recebido terapia antimicrobiana, todos com histórico de pioderma superficial recidivante. A identificação de aproximadamente um quarto de isolados multirresistentes sugere a realização de testes de suscetibilidade 
em todos os casos de pioderma superficial recidivante, pois futuramente, muitos antimicrobianos poderão apresentar-se ineficazes no tratamento das piodermites, assim como de outras doenças que acometem cães. Adicionalmente, as análises bacteriológicas promovem informações que subsidiam a eleição dos antimicrobianos e podem minimizar a ocorrência de resistência bacteriana visto que, conforme exposto, esta tem aumentado nos últimos anos.

\section{CONCLUSÕES}

Pode-se concluir que os isolados de Staphylococcus spp. apresentaram elevada suscetibilidade frente aos antimicrobianos comumente utilizados no tratamento dos piodermas superficiais em cães atendidos no HVU-UFSM, como cefalexina e amoxicilina associada ao ácido clavulânico, confirmando a eleição desses fármacos para o tratamento desta afecção.

A suscetibilidade dos isolados frente às fluoroquinolonas, também recomendadas pela literatura como opção terapêutica nos piodermas, permite sugerir que estes fármacos não devem ser considerados na seleção empírica.

A identificação de Staphylococcus spp. multirresistentes na população canina estudada justifica análises bacteriológicas periódicas e regionais de lesões cutâneas de cães com pioderma superficial, a fim de minimizar a seleção de bactérias resistentes, possíveis falhas terapêuticas e também motiva a antimicrobianoterapia prudente.

\section{REFERÊNCIAS}

Bauer A.W., Kirby W.M., Sherris J.C. \& Turck M. 1966. Antibiotic susceptibility testing by a standardized single disk method. Am. J. Clin. Pathol. 45:493-496.

Bond R. \& Loeffler A. 2012. What's happened to Staphylococcus intermedi$u s$ ? Taxonomic revision and emergence of multi-drug resistence. J. Small Anim. Pract. 56:147-154.

Carter G.R. 1988. Fundamentos de Bacteriologia e Microbiologia Veterinária. $3^{a}$ ed. Roca, São Paulo. 249p.

Cavalcanti S.N. \& Coutinho S.D. 2005. Identificação e perfil de sensibilidade bacteriana de Staphylococcus spp isolados da pele de cães sadios e com piodermite. Clin. Vet. 58:60-66.

CLSI 2006. Performance standards for antimicrobial susceptibility testing. $16^{\text {th }}$ Informational Supplement, Document M100-S16. Wayne, Pennsylvania.

Cox H.U. 2006. Staphylococcal infections, p.316-320. In: Greene C.E. (Ed.), Infectious Disease of the Dog and Cat. $3^{\text {rd }}$ ed. Saunders Elsevier, St Louis. 1387p.

Devriese L.A., Hermans K., Baele M. \& Haesebrouck F. 2009. Staphylococcus pseudintermedius versus Staphylococcus intermedius. Vet. Microbiol. 133:206-207.

Duijkeren E., Catry B., Greko C., Moreno M.A., Pomba M.C., Piörälä S., Ruzauskas M., Sanders P., Threlfa E.J. \& Törneke K. 2011. Review on methicillin-resistant Staphylococcus pseudintermedius. J. Antimicrob. Chemother. 66:2705-2714.

Duquette R.A. \& Nuttall T.J. 2004. Methicillin-resistant Staphylococcus aureus in dogs and cats: an emerging problem? J. Small Anim. Pract. 45:591-597.

Ganiere J.P., Medaille C. \& Mangion C. 2005. Antimicrobial drug susceptibility of Staphylococcus intermedius clinical isolates from canine pyoderma. J. Vet. Med. 52:25-31.

Ghidini F., Piancastelli C., Taddei S., Gandolfo E., Cavirani S. \& Cabassi C.S. 2011. Antibiotic sensitivity of bacterial isolates from cases of canine dermatitis. New Microbiol. 34:403-408.
Gross T.L., Ihrke P.J., Walder E.J. \& Affolter V.K. 2005. Skin diseases of the dog and cat: clinical and histopathologic diagnosis. $2^{\text {nd }}$ ed. Blackwell, Oxford. 932p.

Guardabassi L., Jensen L.B. \& Kruse H. 2008. Guide to Antimicrobial Use in Animals. Blackwell Pusblishing, Oxford. 223p.

Hill P.B. \& Moriello K.A. 1994. Canine pyoderma. J. Am. Vet. Med. Assoc. 204:334-340.

Hill P.B., Eden C.A., Huntley S., Morey V., Ramsey S., Richardson C., Smith D.J., Sutton C., Taylor M.D., Thorpe E., Tidmarsh R. \& Williams V. 2006. Survey of the prevalence, diagnosis and treatment of dermatological conditions in small animals in general practice. Vet. Rec. 158:533539.

Hnilica K.A. \& May E. 2004. Staphylococcal pyoderma: an emerging problem. Compend. Contin. Educ. Pract. Vet. 26:560-568.

Hnilica K.A. 2011. Small Animal Dermatology: a color atlas and therapeutic guide. $3^{\text {rd }}$ ed. W.B. Saunders, Philadelphia. 640p.

Horvath C. \& Neuber A. 2007. Management of canine pyoderma. UK Vet. 12:55-64.

Ihrke J.P. 2005. Management challenges in canine pyoderma. <www.ivis. org/proceedings/navc/2005/.../105. pdf> Acesso em 10 jan. 2013.

Ihrke J.P. 2006. Integumentary infections, p.807-823. In: Greene C.E. (Ed.), Infectious Disease of the Dog and Cat. $3^{\text {rd }}$ ed. Saunders Elsevier, St Louis. $1387 \mathrm{p}$.

Jones R.D., Kania S.A., Rohrbach B.W., Frank L.A. \& Bemis D.A. 2007. Prevalence of oxacilin-and multidrug-resistant staphylococci in clinical samples from dogs: 1772 samples (2001-2005). J. Am. Vet. Med. Assoc. 230:221-227.

Lima L.F.A., Lira A.C., Coutinho H.D.M., Siqueira Junior J.P. \& Barreto H.M. 2012. Antimicrobial resistance in staplylococci isolated from canine pyoderma. Comunicata Scientiae 3:181-185.

Loeffler A., Linek M., Moodley A., Guardabassi L., Sung J.M., Winkler M., Weiss R. \& LIoyd D.H. 2007. First report of multiresistant, mecA-positive Staphylococcus intermedius in Europe: 12 cases from a veterinary dermatology referral clinic in Germany. Vet. Dermatol. 18:412-421.

Lucia M., Moodley A., Latronico F., Giordano A., Caldin M., Fondati A. \& Guardabassi L. 2011. Prevalence of canine methicilin resistant Staphylococcus pseudintermedius in a veterinary diagnostic laboratory in Italy. Res. Vet. Sci. 91:346-348.

Mason I.S. 1991. Canine pyoderma. J. Small Anim.Pract. 32:381-386.

May E.R. 2006. Bacterial skin diseases: current thoughts on pathogenesis and management. Vet. Clin. North Am., Small Anim. Pract. 36:185-202.

Nienhoff U., Kadlec K., Chaberny I.F., Verspohl J., Gerlach G.F., Kreinbrock L., Schwarz S., Simon D. \& Nolte I. 2011. Methicillin-resistant Staphylococcus pseudintermedius among dogs admitted to a small animal hospital. Vet. Microbiol. 150:191-197.

Onuma K., Tanabe T. \& Sato H. 2011. Antimicrobial resistance of Staphylococcus pseudintermedius isolates from healthy dogs and dogs affected with pyoderma in Japan Vet. Dermatol. 23:17-22.

Patel A. \& Forsythe P. 2010. Dermatologia em Pequenos Animais. Elsevier, Rio de Janeiro, p.161-168.

Pedersen K., Pedersen K., Jensen H., Finster K., Jensen V.F. \& Heuer O.E. 2007. Occurrence of antimicrobial resistance in bacteria from diagnostic samples from dogs. J. Antimicrob. Chemother. 60:775-781.

Penna B., Medeiros R., Martins G.M., Martins R.R. \& Lilenbaum W. 2009. In vitro antimicrobial susceptibility of Staphylococci isolated from canine pyoderma in Rio de Janeiro, Brazil. Braz. J. Microbiol. 40:490-494.

Pianta C., Oliveira S.J., Fallavena L.C.B., Esmeraldino A.T. \& Silva Junior V.B. 2006. Pioderma estafilococócico canino: identificação das espécies e sensibilidade aos antimicrobianos. Revta Ciênc. Agrovet. 5:60-63.

Proietti P.C., Bietta A., Coletti M., Marenzoni M.L., Scorza A.V. \& Passamonti F. 2012. Insertion sequence IS256 in canine pyoderma isolates of Staphylococcus pseudintermedius associated with antibiotic resistance. Vet. Microbiol. 157:376-382.

Scott D.W., Miller H.W. \& Griffin C.E. 2001. Muller and Kirk's Small Animal Dermatology. $6^{\text {th }}$ ed. W.B. Saunders, Philadelphia. 1528p. 
Souza T.M., Fighera R.A., Schmidt C., Réquia A.H., Brum J.S., Martins T.B. \& Barros C.S.L. 2009. Prevalência das dermatopatias não-tumorais em cães do município de Santa Maria, Rio Grande do Sul (2005-2008). Pesq. Vet. Bras. 29:157-162.

Spinosa H.S., Górniak S.L. \& Bernardi M.M. 2002. Farmacologia Aplicada à Medicina Veterinária. 3aㅡ ed. Guanabara Koogan, Rio de Janeiro. 752p.

Toma S., Colombo S., Cornegliani L., Persico P., Galzerano M., Gianino M.M. \& Noli C. 2008. Efficacy and tolerability of once-daily cephalexin in canine superficial pyoderma: an open control study. J. Small Anim. Pract. 49:384-391.
Vanni M., Tognetti R., Pretti C., Crema F., Soldani G., Meucci V. \& Intorre L. 2009. Antimicrobial susceptibility of Staphylococcus intermedius and Staphylococcus schleiferi isolated from dogs. Res. Vet. Sci. 87:192-195.

White S.D., Brown A.E., Chapman P.L., Jang S.S. \& Ihrke P.J. 2005. Evaluation of aerobic bacteriologic culture of epidermal collarette specimens in dogs with superficial pyoderma. J. Am. Vet. Medic. Assoc. 6:904-908.

Youn J., Yoon J.W., Koo H.C., Lim S.K. \& Park Y.H. 2011. Prevalence and antimicrogram of Staphylococcus intermedius group isolates from veterinary staff, companion animals, and the environment in veterinary hospitals in Korea. J. Vet. Diagn. Invest. 23:268-274. 